\title{
The Phase Abstraction for Estimating Energy Consumption and Travel Times for Electric Vehicle Route Planning
}

\author{
Payas Rajan \\ payas.rajan@email.ucr.edu \\ Computer Science \& Engineering Department \\ University of California, Riverside \\ USA
}

\author{
Chinya V. Ravishankar \\ ravi@cs.ucr.edu \\ Computer Science \& Engineering Department \\ University of California, Riverside
}

USA

\begin{abstract}
Electric Vehicle (EV) battery capacity is limited, so EV routing must trade off travel time for energy consumption, which grows quadratically with speed. Current multi-parameter EV routing methods assume accurate estimates of time and energy consumed, but current models for obtaining these estimates cannot capture this time-energy tradeoff in a sufficiently flexible way. We present a new approach to EV modeling that addresses such shortcomings. Conventional wisdom holds that models operating at finer time granularities yield better energy consumption estimates. We first show that such is not necessarily the case, by defining a new structuring abstraction for vehicle speed profiles called phases, which models energy consumption accurately at lower temporal granularity. We also address the challenge of generating speed profiles for planned trips with realistic variance in travel times and energy consumed. Our method combines the phase abstraction with Markov chains and kernel density estimation to learn these variations, and construct realistic vehicle speed profiles for real-world routes. Using 52 hours of driving data collected on a Nissan Leaf, we show that our model achieves a per-trip accuracy better than even that of current microscopic models and generates speed profiles that accurately model time and energy consumption at the trip level.
\end{abstract}

\section{CCS CONCEPTS}

- Information systems $\rightarrow$ Global positioning systems; • Applied computing $\rightarrow$ Transportation.

\section{KEYWORDS}

Electric vehicles, energy estimation, route planning

\section{ACM Reference Format:}

Payas Rajan and Chinya V. Ravishankar. 2019. The Phase Abstraction for Estimating Energy Consumption and Travel Times for Electric Vehicle Route Planning. In 27th ACM SIGSPATIAL International Conference on Advances in Geographic Information Systems (SIGSPATIAL '19), November 5-8, 2019, Chicago, IL, USA. ACM, New York, NY, USA, 4 pages. https://doi.org/10. $1145 / 3347146.3359383$

\footnotetext{
Permission to make digital or hard copies of part or all of this work for personal or classroom use is granted without fee provided that copies are not made or distributed for profit or commercial advantage and that copies bear this notice and the full citation on the first page. Copyrights for third-party components of this work must be honored. For all other uses, contact the owner/author(s).

SIGSPATIAL '19, November 5-8, 2019, Chicago, IL, USA

(c) 2019 Copyright held by the owner/author(s).

ACM ISBN 978-1-4503-6909-1/19/11 . \$ \$15.00

https://doi.org/10.1145/3347146.3359383
}

\section{INTRODUCTION}

Electric Vehicle (EV) use is now more widespread than ever, driven both by ecological awareness and strong government incentives. A major constraint, however, is that EVs typically have limited battery capacity, requiring careful energy management. Traditional routing has sought to minimize travel time, assuming that vehicles may travel at the maximum speed allowed by traffic or by law [1]. This is not true for EVs, however, since energy losses from wind resistance grow quadratically with vehicle speed. If initial battery levels are low, raising vehicle speeds to to shorten travel time may also raise battery drain to unacceptable levels. Hence, EV routing must address factors quite different from those for vehicles powered by fossil fuels. The time-energy tradeoff must be made explicitly.

Given its importance, the database community has begun addressing the problem of estimating energy consumption for route planning applications [5, 6], a problem that has historically been addressed by engineers. Also, because the fastest and most energy efficient routes can be very different, there have been efforts to incorporate energy consumption as an explicit metric in route planning [2, 6]. Such energy-aware route planning algorithms require accurate estimates of energy consumption over the network edges or paths. Path-based energy estimates are also useful both for personalized vehicle refueling assistance, and when aggregated, to traffic engineers and policy makers.

\subsection{Our Contributions}

The most important concept we introduce in this work is the abstraction of a phase. Intuitively, a phase is a section of a trip that occurs at an acceleration that is roughly constant. Let $\tau$ be the phase threshold and let the average acceleration of the vehicle be $a$ during some time interval $\left(t_{1}, t_{2}\right)$. We will say that the vehicle is in an acceleration phase during $\left(t_{1}, t_{2}\right)$ if $a>\tau$, a constant speed phase if $-\tau \leq a \leq \tau$, or a deceleration phase if $a<-\tau$. We then use the abstraction of phases to introduce two important models:

- Phase-level EV Energy Estimation Model- We derive an analytic energy consumption model for the three types of phases by integrating the standard analytic microscopic energy consumption model for EVs (see [4]). This aggregation allows us to use phases as the primitive unit of vehicle movement. We show that modeling a vehicle trip as a sequence of phases of discrete average accelerations allows us to estimate the total energy consumption over trips with accuracy superior to even that of microscopic energy consumption models.

- Synthetic trip generation model-In order to be able to prospectively estimate the energy consumption of future trips along a route, we present a model that learns phase transitions and 
phase parameters such as acceleration and phase duration from past trips using Markov chains and kernel density estimation (KDE) respectively. Our model is able to estimate both energy consumption and time taken along future trips along the route with good accuracy. We have evaluated our method with real-life data collected from a 2013 Nissan Leaf driven for 52 hours over a span of five months on 3 different routes in Riverside, California.

\section{RELATED WORK}

Route planning for electric vehicles requires solving two problems: first, accurate information about the energy consumption of EV along the trip must be derived either from historical trips [3], using analytical equations [4], or a combination of both [6]; second, an effective tradeoff between time taken to travel along a path and the energy consumed must be made, which is often solved by energyaware route planning approaches such as [2] to find suitable routes in the underlying road network. Energy estimation can be classified on the basis of their temporal granularity into per-second microscopic models [4], temporally coarser mesoscopic models that use spatial or kinematic parameters to break a trip down into fragments [8], and macroscopic models which use aggregated parameters such as average trip speed. In contrast with standard EV energy estimation models, our model attempts to create a joint model of time taken and energy consumption along a trip.

\section{ESTIMATING EV ENERGY CONSUMPTION WITH PHASES}

We consider vehicle paths on the $X, Y$ plane, where a path $P$ is a sequence of points $\left\{x_{1} x_{2} \ldots x_{n-1} x_{n}\right\}$, with each $x_{i} \in \mathbb{R}^{2}$. We are also given an elevation function $h(x): \mathbb{R}^{2} \rightarrow \mathbb{R}^{+}$that maps each point $x \in \mathbb{R}^{2}$ to its elevation.

Definition 3.1. A speed profile $S=\left\{v_{1} v_{2} \ldots v_{n-1} v_{n}\right\}$ of a vehicle travelling along a route is the sequence of instantaneous speeds $v_{i} \in \mathbb{R}$ of the vehicle at point $x_{i}$.

Definition 3.2. A trip $T=(S, P)$ consists of a speed profile $S$ over a path $P$.

Problem definition: An EV with a given set of vehicle-specific parameters starts from rest at source $s \in \mathbb{R}^{2}$ and travels to a destination $t \in \mathbb{R}^{2}$ along a path $P$ with a speed profile $S$. Our goal is to accurately estimate the time taken and the total energy consumed by EV while travelling along the trip $T=(S, P)$.

\subsection{Instantaneous energy consumption model}

To move the EV along $P$, the vehicle powerplant must do enough work to maintain the vehicle at speed $v(t)$, working against gravity on inclines and overcoming dissipative losses due to rolling friction and wind resistance. The standard approach models these three dissipative forces as follows (see [4], for example).

- Drag force is given by $\left(\frac{\rho A_{f} C_{D}}{2}\right) v^{2}(t)$, where $\rho$ is the air density, $A_{f}$ is the vehicle's aerodynamic cross section and $C_{D}$ is the drag coefficient. For simplicity, we combine the constant terms and define $D=\frac{\rho A_{f} C_{D}}{2 M}$, where $M$ gives the mass of the EV.
- Rolling resistance is given by $N\left(c_{1} v(t)+c_{2}\right)$, where $c_{1}$ and $c_{2}$ are constants and $N=M g \cos \theta(t)$ is the normal reaction of the road surface inclined at angle $\theta(t)$.

- Gravitational resistance to motion is $M g \sin \theta(t)$.

As is common in real EVs, we assume that the vehicle is fitted with regenerative brakes. We account for inefficiencies in the drivetrain and regenerative braking as follows. Let $P_{0}$ be the power that the powerplant must produce if the drivetrain were $100 \%$ efficient, and $P$ be the power that the motor must actually produce to have the same effect on the vehicle. Similarly, let $Q_{0}$ be the energy recovered when the regenerative brakes are $100 \%$ efficient, and $Q$ be the actual power recovered. We define $\eta_{1}=\frac{P}{P_{0}}$ and $\eta_{2}=\frac{Q}{Q_{0}}$. Clearly, $\eta_{1}>0$ and $\eta_{2}<0$.

Since drivetrain and recovery efficiencies are modeled separately, the force to be overcome by the powerplant during acceleration $a(t)$ is

$$
F_{a}(t)=M \eta_{1}\left[a(t)+D v^{2}(t)+g \cos \theta(t)\left(c_{1} v(t)+c_{2}\right)+g \sin \theta(t)\right],
$$

and that to be overcome while decelerating is

$$
F_{d}(t)=M \eta_{2}\left[a(t)+D v^{2}(t)+g \cos \theta(t)\left(c_{1} v(t)+c_{2}\right)+g \sin \theta(t)\right] .
$$

The independent variable in Equations 1 and 2 is the time $t$. We can transform them to make the distance traveled $x$ the independent variable. After the transformation, we get equivalent functions $G_{a}(x)$ and $G_{d}(x)$. We omit the details of this transformation to simplify the exposition. The energy consumed over a trip $T$ of length $d$ is now

$$
E_{I}(T)=\int_{0}^{d} G(x) d x, \text { with } G(x)=\left\{\begin{array}{l}
G_{a}(x) \text { if accelerating } \\
G_{d}(x) \text { if decelerating. }
\end{array}\right.
$$

\subsection{The "phase" abstraction}

We introduce a new structuring abstraction for vehicle trajectories we call phases, and model the EV trip as a sequence of phases. The basic idea is to identify acceleration, deceleration, and constant speed regimes in the given EV trajectory. Consider an EV speed profile $S=\left\{v_{1} v_{2} \ldots v_{N}\right\}$ given as a series of consecutive speeds indexed by time instants. At instant $k$, we say that $\mathrm{EV}$ is accelerating if $v_{k+1} \geq v_{k}$, and decelerating if $v_{k+1}<v_{k}$. We can now assign one of the labels ' $A$ ', ' $D$ ' or ' $R$ ' to each instant, according to whether the vehicle is accelerating, decelerating or at rest. Let the speed profile $S$ generate the label sequence $\lambda_{S}=\left\{L_{1} L_{2} \ldots L_{M}\right\}$ for each instant, where $L_{i} \in\{A, D, R\}$.

We define a run as a series of consecutive of ' $A$ 's or of ' $D$ 's in a label sequence. Given an acceleration threshold $\tau$, we define four types of phases as follows:

Definition 3.3. Acceleration Phase: A maximal run of ' $A$ ' labels during which which the average acceleration exceeds $\tau$.

Definition 3.4. Deceleration Phase: A maximal run of ' $D$ ' labels during which which the average acceleration is lower than $-\tau$.

Definition 3.5. Constant Speed Phase: A maximal run of ' $A$ ' or ' $D$ ' labels where the average acceleration is between $-\tau$ and $\tau$.

Definition 3.6. Rest Phase: A maximal run of ' $R$ ' labels. 
Given an input trip with speed profile $S$ and a phase threshold $\tau$, we finds the runs for which the average acceleration lies in the ranges given in the definitions above. We also partition the speed profile $S$ into a set $\Phi_{A}(S)$ of acceleration phases, a set $\Phi_{D}(S)$ of deceleration phases, a set $\Phi_{C}(S)$ of constant speed phases and a set $\Phi_{R}(S)$ of acceleration phases. Let $\Phi(S)=\Phi_{A}(S) \cup \Phi_{D}(S) \cup \Phi_{C}(S) \cup$ $\Phi_{R}(S)$ be the set of phases for $S$.

\subsection{Energy consumption over a phase}

For each phase, if the EV travels a distance $d$, and we know the force experienced by the EV as a function $G(x)$ of the distance $x$ traveled, we can compute the energy consumption from Equation 3. For a flat road, $\theta \equiv 0$. We define $\beta=g c_{1}, \gamma=g c_{2}$. Let the drivetrain and recovery efficiency factors be $\eta_{1}$ and $\eta_{2}$.

For phase $p$, let $a_{p}$ be the average acceleration (or deceleration), $d_{p}$ be the distance traveled, and $u_{p}$ be the velocity at the start of the phase. Let $E(p)$ denote the energy consumption in phase $p$. Given speed profile $S$, we get upon integrating Equation 3,

$$
E(p)= \begin{cases}M \eta_{1}\left[D u_{p}^{2} d_{p}+\beta u_{p} d_{p}+\gamma d_{p}\right], & \text { if } p \in \Phi_{C}(S) \\ M \eta_{1} E, & \text { if } p \in \Phi_{A}(S) \\ M \eta_{2} E, & \text { if } p \in \Phi_{D}(S) \\ 0, & \text { if } p \in \Phi_{R}(S)\end{cases}
$$

where

$$
E=a_{p} d_{p}+D d_{p}\left(u_{p}^{2}+a_{p} d_{p}\right)+\frac{2 \beta}{3 a_{p}}\left(u_{p}^{2}+2 a_{p} d_{p}\right)^{\frac{3}{2}}+\gamma d_{p} .
$$

When the road is not flat, $\theta \not \equiv 0$, and we must explicitly account for the incline by including the $\sin \theta$ and $\cos \theta$ terms. We find, however, that it is unnecessary to model the effects of gravity in a microscopic manner for each instant, as other models do. Instead, if the end points of a phase $p$ are $q_{i}$ and $q_{j}$, we simply correct for the work $W_{p}=M g\left(h\left(q_{j}\right)-h\left(q_{i}\right)\right)$ done to raise the EV between $q_{i}$ and $q_{j}$.

The energy consumption $E_{\Phi}(T)$ for a trip $T=(S, P)$ is

$$
E_{\Phi}(T)=\sum_{p \in \Phi(S)}\left(E(p)+W_{p}\right)
$$

\subsection{Experimental Validation}

We experimentally evaluated our energy estimation model presented in Section 3.3 by comparing our estimates with real-world energy consumption data. For a detailed description of the postprocessing, see [7]. Next, we compare estimates of our model with that of analytic microscopic energy estimation models of [4], and the observed energy consumption in the EV.

We model each trip as a sequence of phases and use equations 4 and 5 to get $E_{\Phi}(T)$ for all trips $T$. We evaluate our model by comparing its energy estimates with the standard instantaneous model using equation 3 to derive $E_{I}(T)$ for all trips $T$. We use the same parameter values as in [4]. We also verified the performance of both models against the ground truth $E_{M}(T)$, the actual measured energy consumption of the EV. We report the percentage errors ${ }^{1}$ for the estimates of both models in Figure 2.

\footnotetext{
${ }^{1}$ Percentage error is computed as $100 \times\left(\frac{\text { estimate-measured }}{\text { measured }}\right)$.
}

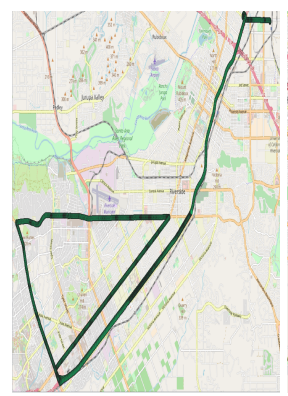

(a) Route 1

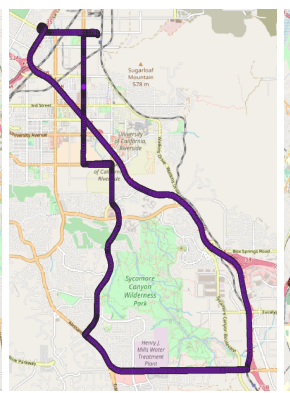

(b) Route 2

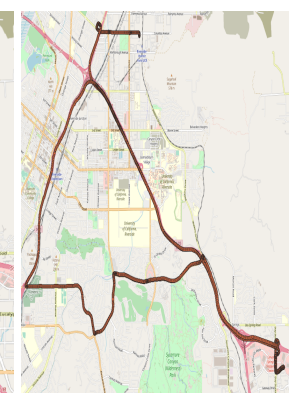

(c) Route 3
Figure 1: Routes over which the EV was driven in Riverside, California. Data from 10 trips along Route 1, 16 trips along Route 2 and 26 trips along Route 3 was collected. Each trip is one hour long.
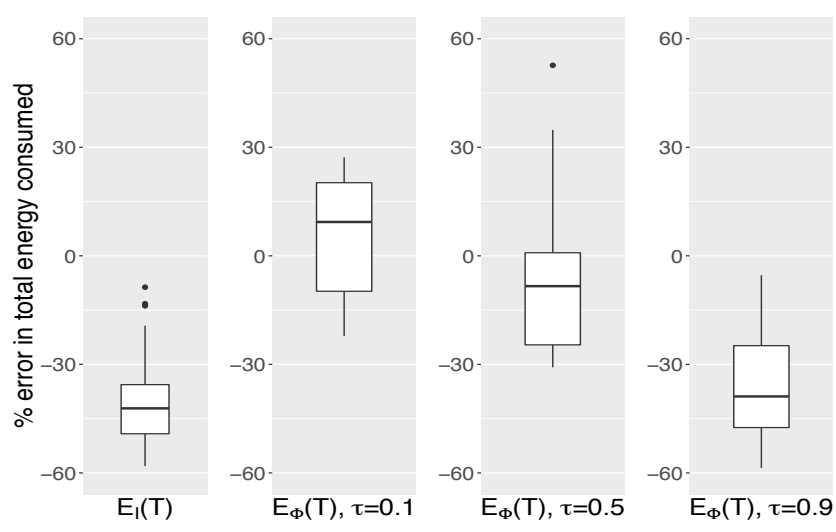

Figure 2: Variance in \% error of $E_{I}(T)$ vs. that of $E_{\Phi}(T)$, with $\tau \in\{0.1,0.5,0.9\}$ for all trips. Negative \% error indicates that the model underestimated energy consumption. Our model performs much better than the standard instantaneous model with percentage errors much closer to 0 .

The box-and-whisker plots in Figure 2 show the percentage error distribution of estimates by the instantaneous model and our model with $\tau \in\{0.1,0.5,0.9\}$. Our model outperforms the instantaneous model, producing percentage errors much closer to zero.

\section{GENERATING REALISTIC SYNTHETIC TRIPS}

Unlike some prior works, our objective is not to produce accurate instantaneous speed profiles. We have shown in Section 3.4 that we might not need speed profiles accurate in this sense, given our objective. Our goal is to generate realistic speed profiles sufficiently close to historical trips to permit accurate time and energy estimates. Thus, our generated speed profiles need not have spatial fidelity over the path $P$. We could generate equivalent vehicle behaviour at a point different from that in input speed profiles and still produce accurate time and energy estimates. 

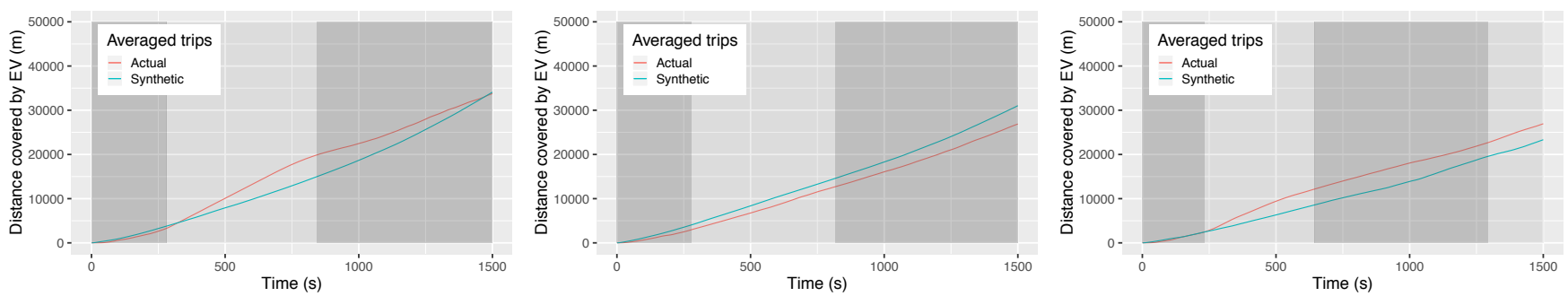

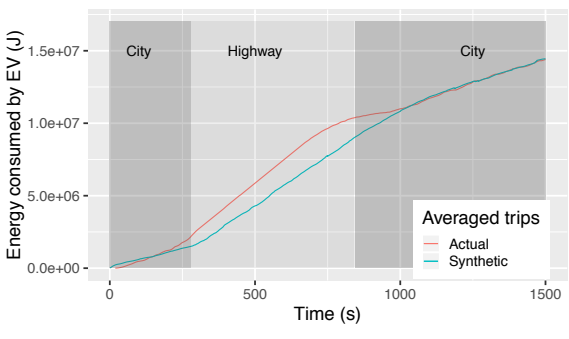

(a) Route 1

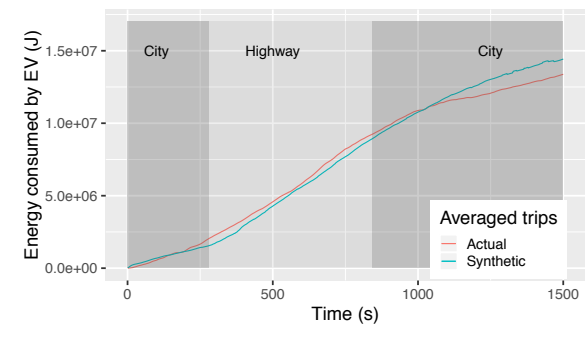

(b) Route 2

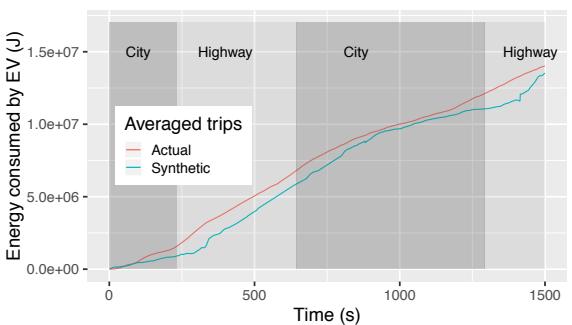

(c) Route 3

Figure 3: Mean distance covered and mean energy consumed by the vehicle as a function of time for observed trips vs a hundred trips generated by our model along the three routes.

As we model each historical trip $T=(S, P)$ as a sequence of phases, we get two outputs: the set of phases $\Phi(S)$, and information about the order in which phases occur in the trip, which is present in the label sequence $\lambda_{S}$. Let the set of label sequences for a set of $N$ historic trips be $\Lambda=\left\{\lambda_{1} \lambda_{2} \ldots \lambda_{N}\right\}$, where $\lambda_{i}$ is the label sequence for the $i^{t h}$ trip. Also, let $\varphi=\left\{\Phi_{1} \Phi_{2} \ldots \Phi_{N}\right\}$ be the set of phases for the $N$ historic trips, where $\Phi_{i}$ is the set of phases in the $i^{t h}$ trip. We show that the information about length and order of phases contained in $\Lambda$ and the acceleration of phases in $\varphi$ is sufficient to produce realistic speed profiles.

Our trip generation model comprises of two components: a fourstate Markov chain that simulates the transitions between different phases as a vehicle moves along a route, and a set of 2-D kernel density estimates accelerations and time duration, one for each type of phase. After the Markov and 2-D KDE models have been prepared, we can generate a synthetic trip along $P$ as follows: Assume that the vehicle always starts at rest and in the acceleration phase. Subsequent phases in the trip are derived from each step of a random walk on the phase transition Markov model starting from the state representing acceleration phases. The random walk gives us the sequence of phases in generated trip with same transition probabilities as past trips. To derive estimates of the length and average acceleration corresponding to each phase of the generated trip, we draw a sample from the 2-D probability distribution estimated in the KDE model for the corresponding type of phase, from which the estimated speed profile $S_{G}$ of the vehicle can then be derived. Since the path $P$ of the generated trip is the same as that of past trips, the final output of the trip generation model is $T_{G}=\left(S_{G}, P\right)$. Figure 3 shows the mean distance travelled and mean energy consumed in all trips over the three routes and a hundred synthetic trips generated by our model with $\tau=0.5$. We observe that distance and energy over time in observed trips is close to that of generated trips.

\section{CONCLUSION}

We showed that using the phase abstraction allows us to accurately estimate energy consumption in EV trips. We also presented a synthetic realistic trip generation method that can be used to accurately model the EV energy consumed in future trips along a route.

\section{ACKNOWLEDGEMENTS}

Thanks are due to G. Wu and M.J. Barth of the Center for Environmental Research and Technology at UC Riverside for the data used in our experiments. This work was supported in part by NSF grant IIS-1527984.

\section{REFERENCES}

[1] Hannah Bast, Daniel Delling, Andrew Goldberg, Matthias Müller-Hannemann, Thomas Pajor, Peter Sanders, Dorothea Wagner, and Renato F Werneck. 2016. Route Planning in Transportation Networks. In Algorithm Engineering. Springer, Cham, 19-80.

[2] Moritz Baum, Julian Dibbelt, Andreas Gemsa, Dorothea Wagner, and Tobias Zündorf. 2015. Shortest Feasible Paths with Charging Stops for Battery Electric Vehicles. In SIGSPATIAL. ACM, New York, NY, USA, 44:1-44:10.

[3] Amir Masoud Bozorgi, Mehdi Farasat, and Anas Mahmoud. 2017. A Time and Energy Efficient Routing Algorithm for Electric Vehicles Based on Historical Driving Data. IEEE Trans. Intell. Vehicles (Dec. 2017), 308-320.

[4] Chiara Fiori, Kyoungho Ahn, and Hesham A Rakha. 2016. Power-based electric vehicle energy consumption model: Model development and validation. Appl. Energy 168 (April 2016), 257-268.

[5] Chenjuan Guo, Bin Yang, Ove Andersen, Christian S Jensen, and Kristian Torp. 2015. EcoMark 2.0: empowering eco-routing with vehicular environmental models and actual vehicle fuel consumption data. Geoinformatica (July 2015), 567-599.

[6] Yan Li, Shashi Shekhar, Pengyue Wang, and William Northrop. 2018. Physicsguided Energy-efficient Path Selection: A Summary of Results. In SIGSPATIAL. ACM, New York, NY, USA, 99-108.

[7] Xuewei Qi, Guoyuan Wu, Kanok Boriboonsomsin, and Matthew J Barth. 2017. Data-driven decomposition analysis and estimation of link-level electric vehicle energy consumption under real-world traffic conditions. Transp. Res. Part D: Trans. Environ. (Aug. 2017)

[8] Ravi Shankar and James Marco. 2013. Method for estimating the energy consumption of electric vehicles and plug-in hybrid electric vehicles under real-world driving conditions. IET Intel. Transport Syst. 7, 1 (March 2013), 138-150. 